\title{
Exact Solutions for Simply Supported and Multilayered Piezothermo- elastic Plates with Imperfect Interfaces
}

\author{
X. Wang ${ }^{*}$ and E. Pan
}

Dept. of Civil Engineering and Dept. of Applied Mathematics, University of Akron, Akron, OH 44325-3905, USA

\begin{abstract}
Exact solutions are derived for three-dimensional, orthotropic, linearly piezothermoelastic, simply-supported, and multilayered rectangular plates with imperfect interfaces under static thermo-electro-mechanical loadings. In this research the imperfect interface is described as thermally weakly (or highly) conducting, mechanically compliant and dielectrically weakly (or highly) conducting. While the homogeneous solutions for one layer are obtained in terms of the socalled pseudo-Stroh formalism, solutions for multilayered plates are expressed in terms of the transfer matrices for both the layer and the imperfect interface. Due to the fact that the thermal effect is incorporated, we adopt a special form of the transfer matrix, resulting in a very concise solution structure for piezothermoelastic multilayered plates. Numerical results are presented to validate the developed formulas and to demonstrate the influence of the interface imperfection on the distributions of the field variables.
\end{abstract}

\section{INTRODUCTION}

Three-dimensional analytical solutions for simplysupported plates continue to attract investigators' attention [1-9]. Various techniques including the asymptotic expansion scheme [7], the state space formulation $[4,7]$, the Stroh formalism [6], the pseudo-Stroh formalism [8,9], and the transfer matrix (or propagator matrix) [2,7-9] have been proposed in these studies. The materials studied encompass purely elastic [1,2], piezoelectric [3,6,7], piezothermoelastic $[4,5]$, and multiferroic $[8,9]$ materials. One common assumption in most of the aforementioned works is that the extended displacement and traction vectors (see [8] for a detailed definition) are continuous across the interface between two adjacent layers. This kind of simplification of the interface is not enough to reflect various damage occurring on the interface (e.g., debonding, sliding and/or cracking across the interface), and as a result the concept of imperfect interface should be incorporated. Up to now various imperfect interface models have been proposed in the context of heat conduction [10-12], dielectricity $[13,14]$ and elasticity [15-20].

The main focus of this research is the incorporation of imperfect interfaces in the three-dimensional analysis of a simply-supported and multilayered piezothermoelastic rectangular plate. The imperfect interface studied here is mechanically compliant and thermally (or dielectrically) weakly (or highly) conducting. For a mechanically compliant interface, we adopt the linear spring model for the imperfect interface [15-19]. In this model, tractions are continuous but displacements are discontinuous across the imperfect interface, jumps in the displacement components are further assumed to be proportional, in terms of the 'spring-factor-type' interface parameters, to their respective interface traction

\footnotetext{
*Address correspondence to this author at the Dept. of Civil Engineering and Dept. of Applied Mathematics, University of Akron, Akron, OH 443253905, USA; E-mail: xuwang@uakron.edu
}

components. For a thermally (or dielectrically) weakly conducting interface [10-13], the normal heat flux (or the normal electric displacement) is continuous but the temperature (or electric potential) is discontinuous across the interface, the jump in temperature (or electric potential) is proportional to the normal heat flux (or normal electric displacement). For a thermally (or dielectrically) highly conducting interface $[11,12,18]$, the temperature (or electric potential) is continuous across the interface whereas the normal heat flux (or normal electric displacement) has a discontinuity across the interface, which is proportional to a certain differential expression of the temperature (or electric potential). It is not a easy task to address this problem since the interface is imperfect in heat conduction, elasticity and dielectricity. Under isothermal conditions, Chen et al. [21] derived exact solutions for simply-supported and multilayered orthotropic piezoelectric rectangular plates with mechanically compliant and dielectrically weakly conducting interface by means of a state space formulation. It should be noticed that in the discussion of Chen et al. [21], there exists a sign error in the description of the dielectrically weakly conducting interface (see Eq. $(11)_{4}$ in [21]).

\section{PROBLEM DESCRIPTION}

\subsection{Basic Equations}

In a fixed Cartesian coordinate system $\left(x_{1}, x_{2}, x_{3}\right)$, the constitutive equations including the Fourier's law of heat conduction for an orthotropic piezothermoelastic material of crystal class $\mathrm{mm} 2$ with poling in the $x_{3}$-direction can be written in the matrix form as

$q_{1}=-k_{11} T_{, 1}, q_{2}=-k_{22} T_{, 2}, q_{3}=-k_{33} T_{, 3}$, 


$$
\left[\begin{array}{l}
\sigma_{11} \\
\sigma_{22} \\
\sigma_{33} \\
\sigma_{23} \\
\sigma_{31} \\
\sigma_{12} \\
D_{1} \\
D_{2} \\
D_{3}
\end{array}\right]=\left[\begin{array}{ccccccccc}
c_{11} & c_{12} & c_{13} & 0 & 0 & 0 & 0 & 0 & e_{31} \\
c_{12} & c_{22} & c_{23} & 0 & 0 & 0 & 0 & 0 & e_{32} \\
c_{13} & c_{23} & c_{33} & 0 & 0 & 0 & 0 & 0 & e_{33} \\
0 & 0 & 0 & c_{44} & 0 & 0 & 0 & e_{24} & 0 \\
0 & 0 & 0 & 0 & c_{55} & 0 & e_{15} & 0 & 0 \\
0 & 0 & 0 & 0 & 0 & c_{66} & 0 & 0 & 0 \\
0 & 0 & 0 & 0 & e_{15} & 0 & -\varepsilon_{11} & 0 & 0 \\
0 & 0 & 0 & e_{24} & 0 & 0 & 0 & -\varepsilon_{22} & 0 \\
e_{31} & e_{32} & e_{33} & 0 & 0 & 0 & 0 & 0 & -\varepsilon_{33}
\end{array}\right]\left[\begin{array}{c}
S_{11} \\
S_{22} \\
S_{33} \\
2 S_{23} \\
2 S_{31} \\
2 S_{12} \\
-E_{1} \\
-E_{2} \\
-E_{3}
\end{array}\right]-\left[\begin{array}{c}
\beta_{11} \\
\beta_{22} \\
\beta_{33} \\
0 \\
0 \\
0 \\
0 \\
0 \\
-p_{3}
\end{array}\right] T
$$

where $q_{i}, \sigma_{i j}$ and $D_{i}$ are the heat flux, stress and electric displacement; $T$ is the temperature change; $S_{i j}$ and $E_{i}$ are the strain and electric field; $k_{i i}$ is the thermal conductivity coefficient; $c_{i j}, e_{i j}$ and $\varepsilon_{i j}$ are the elastic, piezoelectric and dielectric coefficients; $\beta_{i i}$ and $p_{3}$ are the stress-temperature and pyroelectric coefficients. The strains and electric fields can be expressed in terms of the displacements $u_{i}(i=1-3)$ and the electric potential $\phi$ as

$S_{i j}=\frac{1}{2}\left(u_{i, j}+u_{j, i}\right), E_{i}=-\phi_{, i}$.

In addition the steady state energy equation in the absence of heat source and the static equilibrium equations in the absence of body force and electric charge can be expressed as

$q_{i, i}=0$,

$\sigma_{i j, j}=0, D_{i, i}=0$.

\subsection{The Boundary Value Problem}

Let us consider an anisotropic, piezothermoelastic, and $N$-layered rectangular plate with horizontal dimensions $L_{x}, L_{y}$ (in the $x_{1}$ - and $x_{2}$-directions) and the total thickness $H$ (in the $x_{3}$-direction). Each layer of the plate is orthotropic with poling in the $x_{3}$-direction. The Cartesian coordinate system is established in such a way that its origin is at one of the four corners on the bottom surface and the plate is in the positive $x_{3}$ region. Let layer $k$ be bonded by the lower interface $x_{3}=z_{k}$ and the upper interface $x_{3}=z_{k+1}$ with its thickness $h_{k}=z_{k+1}-z_{k}$. It is obvious that $z_{1}=0$ and $z_{N+1}=H$.

The boundary and continuity conditions to be satisfied are those at the four edges of the multilayered rectangular plate, as well as those on the horizontal surfaces and different interfaces of the multilayered rectangular plate. These boundary and continuity conditions are specifically listed as follows

Simply-supported, electrically grounded and zero temperature edge boundary conditions for each layer $[4-8,21]$

$u_{2}=u_{3}=\sigma_{11}=\phi=T=0$ at $x_{1}=0, L_{x}$,
$u_{1}=u_{3}=\sigma_{22}=\phi=T=0$ at $x_{2}=0, L_{y}$, for each layer (ii) Thermo-electro-mechanical boundary conditions on the bottom surface of the plate

$\sigma_{13}=\sigma_{13}^{0} \cos \left(p x_{1}\right) \sin \left(q x_{2}\right)$,

$\sigma_{23}=\sigma_{23}^{0} \sin \left(p x_{1}\right) \cos \left(q x_{2}\right)$,

$\sigma_{33}=\sigma_{33}^{0} \sin \left(p x_{1}\right) \sin \left(q x_{2}\right)$,

$D_{3}=D_{3}^{0} \sin \left(p x_{1}\right) \sin \left(q x_{2}\right)$ or $\phi=\phi^{0} \sin \left(p x_{1}\right) \sin \left(q x_{2}\right)$,

$T_{, 3}-h_{a} T=-h_{a} T_{a}^{*} \sin \left(p x_{1}\right) \sin \left(q x_{2}\right)$,

$\left(x_{3}=0\right)$

where $h_{a}$ is the surface heat transfer coefficient at $x_{3}=0$, and $\sigma_{13}^{0}, \sigma_{23}^{0}, \sigma_{33}^{0}, D_{3}^{0}, \phi^{0}, T_{a}^{*}$ are known values and

$p=\frac{n \pi}{L_{x}}, q=\frac{m \pi}{L_{y}}$,

with $n$ and $m$ being two positive integers.

(iii) Thermo-electro-mechanical boundary conditions on the top surface of the plate

$\sigma_{13}=\sigma_{13}^{H} \cos \left(p x_{1}\right) \sin \left(q x_{2}\right)$,

$\sigma_{23}=\sigma_{23}^{H} \sin \left(p x_{1}\right) \cos \left(q x_{2}\right)$,

$\sigma_{33}=\sigma_{33}^{H} \sin \left(p x_{1}\right) \sin \left(q x_{2}\right)$, $\left(x_{3}=H\right)$

$D_{3}=D_{3}^{H} \sin \left(p x_{1}\right) \sin \left(q x_{2}\right)$ or $\phi=\phi^{H} \sin \left(p x_{1}\right) \sin \left(q x_{2}\right)$,

$T_{, 3}+h_{b} T=h_{b} T_{b}^{*} \sin \left(p x_{1}\right) \sin \left(q x_{2}\right)$

where $h_{b}$ is the surface heat transfer coefficient at $x_{3}=H$, and $\sigma_{13}^{H}, \sigma_{23}^{H}, \sigma_{33}^{H}, D_{3}^{H}, \phi^{H}, T_{b}^{*}$ are also known values.

(iv) Imperfect bonding conditions between two adjacent piezothermoelastic layers

In the following we will discuss in detail the imperfect interface in heat conduction, elasticity and dielectricity one by one.

\section{Imperfect Interface in Heat Conduction}

If the interface $x_{3}=z_{j}(j=2,3, \ldots, N)$ is thermally weakly conducting, then it follows from the definitions outlined in the introduction that the following relationships hold 


$$
\begin{aligned}
& q_{3}\left(x_{1}, x_{2}, z_{j}^{+}\right)=q_{3}\left(x_{1}, x_{2}, z_{j}^{-}\right), \\
& T\left(x_{1}, x_{2}, z_{j}^{+}\right)-T\left(x_{1}, x_{2}, z_{j}^{-}\right)=-\beta_{T}^{(j)} q_{3}\left(x_{1}, x_{2}, z_{j}^{-}\right),
\end{aligned}
$$

where $\beta_{T}^{(j)}$ is a nonnegative constant, and the superscripts "+" and "-" denote the limit values from the upper and lower sides of the interface $z=z_{j}$. The case where $\beta_{T}^{(j)}=0$ corresponds to a thermally perfect interface, whereas $\beta_{T}^{(j)} \rightarrow \infty$ stands for adiabatic contact.

On the other hand, if the interface $x_{3}=z_{j}$ is thermally highly conducting, then it follows from the definitions outlined in the introduction that the following relationships hold

$T\left(x_{1}, x_{2}, z_{j}^{+}\right)=T\left(x_{1}, x_{2}, z_{j}^{-}\right)$,

$q_{3}\left(x_{1}, x_{2}, z_{j}^{+}\right)-q_{3}\left(x_{1}, x_{2}, z_{j}^{-}\right)=\gamma_{T}^{(j)} \Delta_{s} T\left(x_{1}, x_{2}, z_{j}^{-}\right)$,

where $\Delta_{s}=\frac{\partial^{2}}{\partial x_{1}^{2}}+\frac{\partial^{2}}{\partial x_{2}^{2}}$ is the operator of surface Laplacian [18], $\gamma_{T}^{(j)}$ is a nonnegative constant. The case where $\gamma_{T}^{(j)}=0$ corresponds to a thermally perfect interface, whereas $\gamma_{T}^{(j)} \rightarrow \infty$ describes contact with a medium of infinite conductivity.

\section{Imperfect Interface in Elasticity}

According to the definitions outlined in the introduction, the boundary conditions on a mechanically compliant interface $x_{3}=z_{j}$ can be expressed as

$$
\begin{aligned}
& \sigma_{13}\left(x_{1}, x_{2}, z_{j}^{+}\right)=\sigma_{13}\left(x_{1}, x_{2}, z_{j}^{-}\right), \\
& \sigma_{23}\left(x_{1}, x_{2}, z_{j}^{+}\right)=\sigma_{23}\left(x_{1}, x_{2}, z_{j}^{-}\right), \\
& \sigma_{33}\left(x_{1}, x_{2}, z_{j}^{+}\right)=\sigma_{33}\left(x_{1}, x_{2}, z_{j}^{-}\right), \\
& u_{1}\left(x_{1}, x_{2}, z_{j}^{+}\right)-u_{1}\left(x_{1}, x_{2}, z_{j}^{-}\right)=\alpha_{1}^{(j)} \sigma_{13}\left(x_{1}, x_{2}, z_{j}^{-}\right), \\
& u_{2}\left(x_{1}, x_{2}, z_{j}^{+}\right)-u_{2}\left(x_{1}, x_{2}, z_{j}^{-}\right)=\alpha_{2}^{(j)} \sigma_{23}\left(x_{1}, x_{2}, z_{j}^{-}\right), \\
& u_{3}\left(x_{1}, x_{2}, z_{j}^{+}\right)-u_{3}\left(x_{1}, x_{2}, z_{j}^{-}\right)=\alpha_{3}^{(j)} \sigma_{33}\left(x_{1}, x_{2}, z_{j}^{-}\right),
\end{aligned}
$$

where $\alpha_{1}^{(j)}, \alpha_{2}^{(j)}, \alpha_{3}^{(j)}$ are three nonnegative constants. The case where $\alpha_{1}^{(j)}, \alpha_{2}^{(j)}, \alpha_{3}^{(j)}=0$ corresponds to a mechanically perfect interface, whereas $\alpha_{1}^{(j)}, \alpha_{2}^{(j)}, \alpha_{3}^{(j)} \rightarrow \infty$ describes a completely debonded interface.

\section{Imperfect Interface in Dielectricity}

If the interface $x_{3}=z_{j}$ is dielectrically weakly conducting, then the following relationships hold

$$
\begin{aligned}
& D_{3}\left(x_{1}, x_{2}, z_{j}^{+}\right)=D_{3}\left(x_{1}, x_{2}, z_{j}^{-}\right), \\
& \phi\left(x_{1}, x_{2}, z_{j}^{+}\right)-\phi\left(x_{1}, x_{2}, z_{j}^{-}\right)=-\beta_{D}^{(j)} D_{3}\left(x_{1}, x_{2}, z_{j}^{-}\right),
\end{aligned}
$$

where $\beta_{D}^{(j)}$ is a nonnegative constant. The case where $\beta_{D}^{(j)}=0$ corresponds to a dielectrically perfect interface, whereas $\beta_{D}^{(j)} \rightarrow \infty$ describes a charge-free (insulating) interface.

On the other hand, if the interface $x_{3}=z_{j}$ is dielectrically highly conducting, then the following relationships hold

$$
\begin{aligned}
& \phi\left(x_{1}, x_{2}, z_{j}^{+}\right)=\phi\left(x_{1}, x_{2}, z_{j}^{-}\right), \\
& D_{3}\left(x_{1}, x_{2}, z_{j}^{+}\right)-D_{3}\left(x_{1}, x_{2}, z_{j}^{-}\right)=\gamma_{D}^{(j)} \Delta_{s} \phi\left(x_{1}, x_{2}, z_{j}^{-}\right),
\end{aligned}
$$

where $\gamma_{D}^{(j)}$ is a nonnegative constant. The case where $\gamma_{D}^{(j)}=0$ corresponds to a dielectrically perfect interface, whereas $\gamma_{D}^{(j)} \rightarrow \infty$ describes an equipotential interface.

\section{THE PSEUDO-STROH FORMALISM}

\subsection{Heat Conduction}

The temperature $T$ satisfying the zero temperature edge conditions is chosen to be

$$
T=f e^{\eta x_{3}} \sin \left(p x_{1}\right) \sin \left(q x_{2}\right) .
$$

where $f$ and $\eta$ are unknowns.

Then it follows from Eq. (1) that the three heat flux components can be expressed as

$q_{1}=h_{1} e^{\eta x_{3}} \cos \left(p x_{1}\right) \sin \left(q x_{2}\right), q_{2}=h_{2} e^{\eta x_{3}}$

$\times \sin \left(p x_{1}\right) \cos \left(q x_{2}\right), q_{3}=g e^{\eta x_{3}} \sin \left(p x_{1}\right) \sin \left(q x_{2}\right)$,

where $h_{1}=-k_{11} p f, h_{2}=-k_{22} q f, g=-k_{33} \eta f$.

Substitution of Eq. (15) into Eq. (1), then the results into Eq. (4) for the energy equation, we obtain the following eigenrelation

$$
\left(\eta^{2}-\frac{k_{11} p^{2}+k_{22} q^{2}}{k_{33}}\right) f=0
$$

It is apparent that for a nontrivial solution of $f$, the two eigenvalues of $\eta$ should be

$\eta_{1}=\sqrt{\frac{k_{11} p^{2}+k_{22} q^{2}}{k_{33}}}>0, \quad \eta_{2}=-\eta_{1}<0$.

With the aid of Eq. (16), Eq. (17) can be recast into the following standard eigenvalue problem

$$
\mathbf{M}\left[\begin{array}{l}
f \\
g
\end{array}\right]=\eta\left[\begin{array}{l}
f \\
g
\end{array}\right],
$$

where

$$
\mathbf{M}=-\left[\begin{array}{cc}
0 & 1 / k_{33} \\
k_{11} p^{2}+k_{22} q^{2} & 0
\end{array}\right] .
$$

If we distinguish the two eigenvectors of Eq. (19) by attaching a subscript to $f$ and $g$, then the general solution for the temperature and normal heat flux (of the $x_{3}$-dependent factor) can be expressed as 
$\left[\begin{array}{l}T \\ q_{3}\end{array}\right]=\left[\begin{array}{ll}f_{1} & f_{2} \\ g_{1} & g_{2}\end{array}\right]\left[\begin{array}{cc}e_{1}^{\eta_{1} x_{3}} & 0 \\ 0 & e^{-\eta_{1} x_{3}}\end{array}\right]\left[\begin{array}{l}\chi_{1} \\ \chi_{2}\end{array}\right]$

where $\chi_{1}$ and $\chi_{2}$ are two unknown constants to be determined. The two in-plane heat fluxes $q_{1}$ and $q_{2}$ (of the $x_{3}$ dependent factor) can be expressed in terms of the temperature and normal heat flux as

$\left[\begin{array}{l}q_{1} \\ q_{2}\end{array}\right]=-\left[\begin{array}{ll}k_{11} p & 0 \\ k_{22} q & 0\end{array}\right]\left[\begin{array}{l}T \\ q_{3}\end{array}\right]$.

The eigenvectors of Eq. (19) are actually right ones. The left eigenvectors of Eq. (19) are found by solving the following eigenvalue problem

$\mathbf{M}^{T} \boldsymbol{\delta}=\lambda \boldsymbol{\delta}$,

where the superscript $T$ denotes matrix transpose.

If $\eta$ and $[f, g]^{T}$ are the eigenvalue and eigenvector of Eq. (19), then $\lambda=-\eta$ and $\delta=[-g, f]^{T}$ are the corresponding solutions of Eq. (23). Since the left and right eigenvectors are orthogonal to each other, we then come to the following normalized orthogonal relationship

$\left[\begin{array}{cc}-g_{2} & f_{2} \\ g_{1} & -f_{1}\end{array}\right]\left[\begin{array}{ll}f_{1} & f_{2} \\ g_{1} & g_{2}\end{array}\right]=\left[\begin{array}{ll}1 & 0 \\ 0 & 1\end{array}\right]$.

The choice of $f_{1}, f_{2}, g_{1}$ and $g_{2}$ is not unique as long as they satisfy Eq. (24) or equivalently $f_{1} f_{2}=-1 /\left(2 k_{33} \eta_{1}\right)$. For example we can choose $f_{1}=1, f_{2}=-1 /\left(2 k_{33} \eta_{1}\right), g_{1}=-k_{33} \eta_{1}, g_{2}=-1 / 2$, which obviously satisfy the above normalized orthogonal relationship.

\subsection{The Electroelastic Field}

In view of the simply supported and electrically grounded edge boundary conditions in Eq. (6), the generalized displacement vector can take the following forms

$$
\begin{aligned}
& \mathbf{u}=\left[\begin{array}{l}
u_{1} \\
u_{2} \\
u_{3} \\
\phi
\end{array}\right]=e^{s x_{3}}\left[\begin{array}{l}
a_{1} \cos \left(p x_{1}\right) \sin \left(q x_{2}\right) \\
a_{2} \sin \left(p x_{1}\right) \cos \left(q x_{2}\right) \\
a_{3} \sin \left(p x_{1}\right) \sin \left(q x_{2}\right) \\
a_{4} \sin \left(p x_{1}\right) \sin \left(q x_{2}\right)
\end{array}\right] \\
& +f e^{\eta x_{3}}\left[\begin{array}{l}
c_{1} \cos \left(p x_{1}\right) \sin \left(q x_{2}\right) \\
c_{2} \sin \left(p x_{1}\right) \cos \left(q x_{2}\right) \\
c_{3} \sin \left(p x_{1}\right) \sin \left(q x_{2}\right) \\
c_{4} \sin \left(p x_{1}\right) \sin \left(q x_{2}\right)
\end{array}\right],
\end{aligned}
$$

where the first term on the right hand side of Eq. (25) gives the homogeneous general solution, while the second term on the right hand side of Eq. (25) presents the particular solution due to the thermal effect from Eq. (15).

Substitution of Eq. (25) into Eq. (3), and then the results into the constitutive relations (2) yields the generalized traction vector as follows

$$
\begin{aligned}
& \mathbf{t}=\left[\begin{array}{l}
\sigma_{13} \\
\sigma_{23} \\
\sigma_{33} \\
D_{3}
\end{array}\right]=e^{s x_{3}}\left[\begin{array}{l}
b_{1} \cos \left(p x_{1}\right) \sin \left(q x_{2}\right) \\
b_{2} \sin \left(p x_{1}\right) \cos \left(q x_{2}\right) \\
b_{3} \sin \left(p x_{1}\right) \sin \left(q x_{2}\right) \\
b_{4} \sin \left(p x_{1}\right) \sin \left(q x_{2}\right)
\end{array}\right] \\
& +f e^{\eta x_{3}}\left[\begin{array}{l}
d_{1} \cos \left(p x_{1}\right) \sin \left(q x_{2}\right) \\
d_{2} \sin \left(p x_{1}\right) \cos \left(q x_{2}\right) \\
d_{3} \sin \left(p x_{1}\right) \sin \left(q x_{2}\right) \\
d_{4} \sin \left(p x_{1}\right) \sin \left(q x_{2}\right)
\end{array}\right] .
\end{aligned}
$$

Introducing four vectors $\mathbf{a}, \mathbf{b}, \mathbf{c}, \mathbf{d}$ of dimension 4

$$
\begin{array}{lll}
\mathbf{a}=\left[\begin{array}{llll}
a_{1} & a_{2} & a_{3} & a_{4}
\end{array}\right]^{T}, & \mathbf{b}=\left[\begin{array}{llll}
b_{1} & b_{2} & b_{3} & b_{4}
\end{array}\right]^{T}, \\
\mathbf{c}=\left[\begin{array}{llll}
c_{1} & c_{2} & c_{3} & c_{4}
\end{array}\right]^{T}, \quad \mathbf{d}=\left[\begin{array}{llll}
d_{1} & d_{2} & d_{3} & d_{4}
\end{array}\right]^{T},
\end{array}
$$

then we can find that the vector $\mathbf{b}$ is related to $\mathbf{a}$, and $\mathbf{d}$ to $\mathbf{c}$ by

$$
\begin{aligned}
& \mathbf{b}=\left(\mathbf{R}^{\prime}+s \mathbf{T}\right) \mathbf{a}=-\frac{1}{s}(\mathbf{Q}+s \mathbf{R}) \mathbf{a}, \\
& \mathbf{d}=\left(\mathbf{R}^{\prime}+\eta \mathbf{T}\right) \mathbf{c}-\boldsymbol{\gamma}_{2}=-\frac{1}{\eta}(\mathbf{Q}+\eta \mathbf{R}) \mathbf{c}+\frac{1}{\eta} \boldsymbol{\gamma}_{1} .
\end{aligned}
$$

where $\mathbf{R}^{\prime}=-\mathbf{R}^{T}$, and the three $4 \times 4$ real matrices $\mathbf{T}, \mathbf{Q}, \mathbf{R}$ are defined by

$$
\begin{aligned}
& \mathbf{T}=\mathbf{T}^{T}=\left[\begin{array}{cccc}
c_{55} & 0 & 0 & 0 \\
0 & c_{44} & 0 & 0 \\
0 & 0 & c_{33} & e_{33} \\
0 & 0 & e_{33} & -\varepsilon_{33}
\end{array}\right], \\
& \mathbf{Q}=\mathbf{Q}^{T}=\left[\begin{array}{cccc}
-\left(c_{11} p^{2}+c_{66} q^{2}\right) & -p q\left(c_{12}+c_{66}\right) & 0 & 0 \\
-p q\left(c_{12}+c_{66}\right) & -\left(c_{66} p^{2}+c_{22} q^{2}\right) & 0 & 0 \\
0 & 0 & -\left(c_{55} p^{2}+c_{44} q^{2}\right) & -\left(e_{15} p^{2}+e_{24} q^{2}\right) \\
0 & 0 & -\left(e_{15} p^{2}+e_{24} q^{2}\right) & \varepsilon_{11} p^{2}+\varepsilon_{22} q^{2}
\end{array}\right] \\
& \mathbf{R}=\left[\begin{array}{cccc}
0 & 0 & p c_{13} & p e_{31} \\
0 & 0 & q c_{23} & q e_{32} \\
-p c_{55} & -q c_{44} & 0 & 0 \\
-p e_{15} & -q e_{24} & 0 & 0
\end{array}\right],
\end{aligned}
$$

and the two real vectors $\gamma_{1}, \gamma_{2}$ are defined as

$\boldsymbol{\gamma}_{1}=\left[\begin{array}{c}p \beta_{11} \\ q \beta_{22} \\ 0 \\ 0\end{array}\right], \gamma_{2}=\left[\begin{array}{c}0 \\ 0 \\ \beta_{33} \\ -p_{3}\end{array}\right]$,

In addition, the in-plane stresses and the in-plane electric displacements can be expressed as

$\left[\begin{array}{l}\sigma_{11} \\ \sigma_{12} \\ \sigma_{22} \\ D_{1} \\ D_{2}\end{array}\right]=e^{s x_{3}}\left[\begin{array}{c}q_{11} \sin \left(p x_{1}\right) \sin \left(q x_{2}\right) \\ q_{21} \cos \left(p x_{1}\right) \cos \left(q x_{2}\right) \\ q_{31} \sin \left(p x_{1}\right) \sin \left(q x_{2}\right) \\ q_{41} \cos \left(p x_{1}\right) \sin \left(q x_{2}\right) \\ q_{51} \sin \left(p x_{1}\right) \cos \left(q x_{2}\right)\end{array}\right]+f e^{\eta x_{3}}\left[\begin{array}{c}q_{12} \sin \left(p x_{1}\right) \sin \left(q x_{2}\right) \\ q_{22} \cos \left(p x_{1}\right) \cos \left(q x_{2}\right) \\ q_{32} \sin \left(p x_{1}\right) \sin \left(q x_{2}\right) \\ q_{42} \cos \left(p x_{1}\right) \sin \left(q x_{2}\right) \\ q_{52} \sin \left(p x_{1}\right) \cos \left(q x_{2}\right)\end{array}\right]$,

where

$\left[\begin{array}{l}q_{11} \\ q_{21} \\ q_{31} \\ q_{41} \\ q_{51}\end{array}\right]=\left[\begin{array}{cccc}-c_{11} p & -c_{12} q & c_{13} s & e_{31} s \\ c_{66} q & c_{66} p & 0 & 0 \\ -c_{12} p & -c_{22} q & c_{23} s & e_{32} s \\ e_{15} s & 0 & e_{15} p & -\varepsilon_{11} p \\ 0 & e_{24} s & e_{24} q & -\varepsilon_{22} q\end{array}\right]\left[\begin{array}{l}a_{1} \\ a_{2} \\ a_{3} \\ a_{4}\end{array}\right]$, 


$$
\left[\begin{array}{l}
q_{12} \\
q_{22} \\
q_{32} \\
q_{42} \\
q_{52}
\end{array}\right]=\left[\begin{array}{cccc}
-c_{11} p & -c_{12} q & c_{13} \eta & e_{31} \eta \\
c_{66} q & c_{66} p & 0 & 0 \\
-c_{12} p & -c_{22} q & c_{23} \eta & e_{32} \eta \\
e_{15} \eta & 0 & e_{15} p & -\varepsilon_{11} p \\
0 & e_{24} \eta & e_{24} q & -\varepsilon_{22} q
\end{array}\right]\left[\begin{array}{c}
c_{1} \\
c_{2} \\
c_{3} \\
c_{4}
\end{array}\right]-\left[\begin{array}{c}
\beta_{11} \\
0 \\
\beta_{22} \\
0 \\
0
\end{array}\right],
$$

Now inserting Eq. (25) into Eq. (3), then into Eq. (2), and finally into the static equilibrium equations (5), we finally arrive at the following eigenrelations

$\left[\mathbf{Q}+s\left(\mathbf{R}+\mathbf{R}^{\prime}\right)+s^{2} \mathbf{T}\right] \mathbf{a}=\mathbf{0}$,

$\left[\mathbf{Q}+\eta\left(\mathbf{R}+\mathbf{R}^{\prime}\right)+\eta^{2} \mathbf{T}\right] \mathbf{c}=\boldsymbol{\gamma}_{1}+\eta \boldsymbol{\gamma}_{2}$.

It can be easily verified that if $s$ is an eigenvalue of (34), then $-S$ is also an eigenvalue of the eigenequation (34). With aid of Eqs. (28) and (29), Eqs. (34) and (35) can be recast into the following standard eigenrelations

$\mathbf{N}\left[\begin{array}{l}\mathbf{a} \\ \mathbf{b}\end{array}\right]=s\left[\begin{array}{l}\mathbf{a} \\ \mathbf{b}\end{array}\right]$,

$\mathbf{N}\left[\begin{array}{l}\mathbf{c} \\ \mathbf{d}\end{array}\right]=\eta\left[\begin{array}{l}\mathbf{c} \\ \mathbf{d}\end{array}\right]+\boldsymbol{\gamma}$

where

$\mathbf{N}=\left[\begin{array}{cc}-\mathbf{T}^{-1} \mathbf{R}^{\prime} & \mathbf{T}^{-1} \\ -\mathbf{Q}+\mathbf{R} \mathbf{T}^{-1} \mathbf{R}^{\prime} & -\mathbf{R} \mathbf{T}^{-1}\end{array}\right], \boldsymbol{\gamma}=-\left[\begin{array}{cc}\mathbf{0} & \mathbf{T}^{-1} \\ \mathbf{I} & -\mathbf{R} \mathbf{T}^{-1}\end{array}\right]\left[\begin{array}{l}\gamma_{1} \\ \gamma_{2}\end{array}\right]$.

The general solutions Eqs. (36) and (37) can be termed the pseudo-Stroh formalism [8] for a homogeneous piezothermoelastic layer. Assume that the $i$ th $(i=1-4)$ and $(i+4)$ th eigenvalues of Eq. (36), denoted by $s_{i}$ and $s_{i+4}$, satisfy the relation $s_{i}+s_{i+4}=0$. We distinguish the eight eigenvectors of Eq. (36) by attaching a subscript to a and b. Also $\left[\begin{array}{ll}\mathbf{c}_{1}^{T} & \mathbf{d}_{1}^{T}\end{array}\right]^{T}$ is the vector obtained from Eq. (37) for $\eta=\eta_{1}$, $\left[\begin{array}{ll}\mathbf{c}_{2}^{T} & \mathbf{d}_{2}^{T}\end{array}\right]^{T}$ is the vector obtained from Eq. (37) for $\eta=\eta_{2}$.

Then the general solution for the generalized displacement and traction vectors (of the $x_{3}$-dependent factor) can be concisely expressed as

$\left[\begin{array}{l}\mathbf{u} \\ \mathbf{t}\end{array}\right]=\left[\begin{array}{ll}\mathbf{A}_{1} & \mathbf{A}_{2} \\ \mathbf{B}_{1} & \mathbf{B}_{2}\end{array}\right]\left\langle e^{s_{\alpha} x_{3}}\right\rangle\left[\begin{array}{l}\mathbf{K}_{1} \\ \mathbf{K}_{2}\end{array}\right]+\left[\begin{array}{cc}\mathbf{c}_{1} & \mathbf{c}_{2} \\ \mathbf{d}_{1} & \mathbf{d}_{2}\end{array}\right]\left[\begin{array}{cc}\eta_{1} x_{3} & 0 \\ 0 & e^{-\eta_{1} x_{3}}\end{array}\right]\left[\begin{array}{cc}f_{1} & 0 \\ 0 & f_{2}\end{array}\right]\left[\begin{array}{l}\chi_{1} \\ \chi_{2}\end{array}\right]$,

where $\mathbf{K}_{1}$ and $\mathbf{K}_{2}$ are two constant vectors to be determined, and

$\mathbf{A}_{1}=\left[\begin{array}{llll}\mathbf{a}_{1} & \mathbf{a}_{2} & \mathbf{a}_{3} & \mathbf{a}_{4}\end{array}\right], \quad \mathbf{A}_{2}=\left[\begin{array}{llll}\mathbf{a}_{5} & \mathbf{a}_{6} & \mathbf{a}_{7} & \mathbf{a}_{8}\end{array}\right]$,

$\mathbf{B}_{1}=\left[\begin{array}{llll}\mathbf{b}_{1} & \mathbf{b}_{2} & \mathbf{b}_{3} & \mathbf{b}_{4}\end{array}\right], \quad \mathbf{B}_{2}=\left[\begin{array}{llll}\mathbf{b}_{5} & \mathbf{b}_{6} & \mathbf{b}_{7} & \mathbf{b}_{8}\end{array}\right]$,

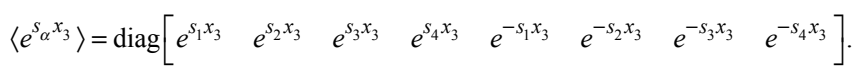

In view of Eqs. (21) and (24), Eq. (39) can also be further expressed as

$$
\begin{aligned}
& {\left[\begin{array}{l}
\mathbf{u} \\
\mathbf{t}
\end{array}\right]=\left[\begin{array}{ll}
\mathbf{A}_{1} & \mathbf{A}_{2} \\
\mathbf{B}_{1} & \mathbf{B}_{2}
\end{array}\right]\left\langle e^{\left.s_{\alpha} x_{3}\right\rangle}\left[\begin{array}{l}
\mathbf{K}_{1} \\
\mathbf{K}_{2}
\end{array}\right]+\right.} \\
& {\left[\begin{array}{ll}
\mathbf{c}_{1} & \mathbf{c}_{2} \\
\mathbf{d}_{1} & \mathbf{d}_{2}
\end{array}\right]\left[\begin{array}{cc}
-f_{1} g_{2} & f_{1} f_{2} \\
f_{2} g_{1} & -f_{1} f_{2}
\end{array}\right]\left[\begin{array}{l}
T \\
q_{3}
\end{array}\right] .}
\end{aligned}
$$

The eigenvectors of Eq. (36) are actually right ones. The left eigenvectors of Eq. (36) are found by solving the following eigenvalue problem

$\mathbf{N}^{T} \eta=\lambda \eta$

It can be shown that if $s$ and $[\mathbf{a}, \mathbf{b}]^{T}$ are the eigenvalue and eigenvector of Eq. (36), then $\lambda=-s$ and $\eta=[-\mathbf{b}, \mathbf{a}]^{T}$ are the corresponding solutions of Eq. (42). Since the left and right eigenvectors are orthogonal to each other, we then come to the following important orthogonal relationship [8]

$\left[\begin{array}{cc}-\mathbf{B}_{2}^{T} & \mathbf{A}_{2}^{T} \\ \mathbf{B}_{1}^{T} & -\mathbf{A}_{1}^{T}\end{array}\right]\left[\begin{array}{ll}\mathbf{A}_{1} & \mathbf{A}_{2} \\ \mathbf{B}_{1} & \mathbf{B}_{2}\end{array}\right]=\left[\begin{array}{ll}\mathbf{I} & \mathbf{0} \\ \mathbf{0} & \mathbf{I}\end{array}\right]$

Thus the orthogonal relationship Eq. (43) provides us with a simple way of inverting the eigenvector matrix, which is required in forming the transfer matrix.

Furthermore after tedious derivations, the in-plane stresses, electric displacements and heat fluxes (of the $x_{3}$ dependent factor) can be expressed in terms of the generalized displacement vector, traction vector, temperature and normal heat flux as

$$
\left[\begin{array}{c}
\sigma_{11} \\
\sigma_{12} \\
\sigma_{22} \\
D_{1} \\
D_{2} \\
q_{1} \\
q_{2}
\end{array}\right]=\left[\begin{array}{cccccccccc}
p \mathrm{M}_{11} & q \mathrm{M}_{12} & 0 & 0 & 0 & 0 & \mathrm{M}_{17} & \mathrm{M}_{18} & \mathrm{M}_{19} & 0 \\
q \mathrm{M}_{21} & p \mathrm{M}_{21} & 0 & 0 & 0 & 0 & 0 & 0 & 0 & 0 \\
p \mathrm{M}_{12} & q \mathrm{M}_{32} & 0 & 0 & 0 & 0 & \mathrm{M}_{37} & \mathrm{M}_{38} & \mathrm{M}_{39} & 0 \\
0 & 0 & 0 & p \mathrm{M}_{44} & \mathrm{M}_{45} & 0 & 0 & 0 & 0 & 0 \\
0 & 0 & 0 & q \mathrm{M}_{54} & 0 & \mathrm{M}_{56} & 0 & 0 & 0 & 0 \\
0 & 0 & 0 & 0 & 0 & 0 & 0 & 0 & p \mathrm{M}_{69} & 0 \\
0 & 0 & 0 & 0 & 0 & 0 & 0 & 0 & q \mathrm{M}_{79} & 0
\end{array}\right]\left[\begin{array}{c}
\mathbf{u} \\
\mathbf{t} \\
T \\
q_{3}
\end{array}\right],(
$$

where $\mathbf{M}_{i j}$ are only related to the material properties given by

$$
\begin{aligned}
& \mathrm{M}_{11}=\frac{c_{13}^{2} \varepsilon_{33}+2 c_{13} e_{33} e_{31}-c_{33} e_{31}^{2}}{c_{33} \varepsilon_{33}+e_{33}^{2}}-c_{11}, \\
& \mathrm{M}_{12}=\frac{c_{13} c_{23} \varepsilon_{33}+c_{13} e_{33} e_{32}+c_{23} e_{33} e_{31}-c_{33} e_{31} e_{32}}{c_{33} \varepsilon_{33}+e_{33}^{2}}-c_{12}, \\
& \mathrm{M}_{17}=\frac{c_{13} \varepsilon_{33}+e_{31} e_{33}}{c_{33} \varepsilon_{33}+e_{33}^{2}}, \mathrm{M}_{18}=\frac{c_{13} e_{33}-c_{33} e_{31}}{c_{33} \varepsilon_{33}+e_{33}^{2}}, \\
& \mathrm{M}_{19}=\frac{c_{13}\left(\varepsilon_{33} \beta_{33}-e_{33} p_{3}\right)+e_{31}\left(e_{33} \beta_{33}+c_{33} p_{3}\right)}{c_{33} \varepsilon_{33}+e_{33}^{2}}-\beta_{11}, \\
& \mathrm{M}_{21}=c_{66}, \\
& \mathrm{M}_{32}=\frac{c_{23}^{2} \varepsilon_{33}+2 c_{23} e_{33} e_{32}-c_{33} e_{32}^{2}}{c_{33} \varepsilon_{33}+e_{33}^{2}}-c_{22}, \mathrm{M}_{37}=\frac{c_{23} \varepsilon_{33}+e_{32} e_{33}}{c_{33} \varepsilon_{33}+e_{33}^{2}}, \\
& \mathrm{M}_{38}=\frac{c_{23} e_{33}-c_{33} e_{32}}{c_{33} \varepsilon_{33}+e_{33}^{2}}, \mathrm{M}_{39}=\frac{c_{23}\left(\varepsilon_{33} \beta_{33}-e_{33} p_{3}\right)+e_{32}\left(e_{33} \beta_{33}+c_{33} p_{3}\right)}{c_{33} \varepsilon_{33}+e_{33}^{2}}-\beta_{22}, \\
& \mathrm{M}_{44}=-\frac{c_{55} \varepsilon_{11}+e_{15}^{2}}{c_{55}}, \mathrm{M}_{45}=\frac{e_{15}}{c_{55}}, \mathrm{M}_{54}=-\frac{c_{44} \varepsilon_{22}+e_{24}^{2}}{c_{44}}, \mathrm{M}_{56}=\frac{e_{24}}{c_{44}}, \mathrm{M}_{69}=-k_{11}, \quad \mathrm{M}_{79}=-k_{22} .
\end{aligned}
$$

The above expression Eq. (44) demonstrates that once the generalized displacement vector, generalized traction vector, temperature and normal heat flux are known, all the other inplane field components can be easily obtained through an algebraic operation.

\section{TRANSFER MATRIX AND SOLUTION OF LAY- ERED STRUCTURE}

For a certain layer $k$ with its lower surface at $x_{3}=z_{k}^{+}$ ( $k=1,2, \cdots, N)$, it follows from Eqs. (21) and (24) that $\chi_{1}$ and $\chi_{2}$ can be expressed in terms of temperature and normal heat flux at its lower surface $x_{3}=z_{k}^{+}$as 


$$
\begin{aligned}
& {\left[\begin{array}{l}
\chi_{1} \\
\chi_{2}
\end{array}\right]=\left[\begin{array}{cc}
e^{-\eta_{1} z_{k}} & 0 \\
0 & e^{\eta_{1} z_{k}}
\end{array}\right]\left[\begin{array}{ll}
f_{1} & f_{2} \\
g_{1} & g_{2}
\end{array}\right]^{-1}\left[\begin{array}{c}
T \\
q_{3}
\end{array}\right]_{z_{k}^{+}}} \\
& =\left[\begin{array}{cc}
e^{-\eta_{1} z_{k}} & 0 \\
0 & e^{\eta_{1} z_{k}}
\end{array}\right]\left[\begin{array}{cc}
-g_{2} & f_{2} \\
g_{1} & -f_{1}
\end{array}\right]\left[\begin{array}{c}
T \\
q_{3}
\end{array}\right]_{z_{k}^{+}} .
\end{aligned}
$$

Then the temperature and normal heat flux at any position within this layer are related to those at its lower surface $x_{3}=z_{k}^{+}$as follows

$\left[\begin{array}{c}T \\ q_{3}\end{array}\right]=\mathbf{P}_{k}\left(x_{3}-z_{k}\right)\left[\begin{array}{c}T \\ q_{3}\end{array}\right]_{z_{k}^{+}}$,

where

$\mathbf{P}_{k}\left(x_{3}\right)=\left[\begin{array}{cc}f_{2} g_{1} e^{-\eta_{1} x_{3}}-f_{1} g_{2} e^{\eta_{1} x_{3}} & f_{1} f_{2}\left(e^{\eta_{1} x_{3}}-e^{-\eta_{1} x_{3}}\right) \\ g_{1} g_{2}\left(e^{-\eta_{1} x_{3}}-e^{\eta_{1} x_{3}}\right) & f_{2} g_{1} e^{\eta_{1} x_{3}}-f_{1} g_{2} e^{-\eta_{1} x_{3}}\end{array}\right]$,

is the transfer matrix for heat conduction of layer $k$. Apparently $\mathbf{P}_{k}(0)$ is a $2 \times 2$ identity matrix.

Similarly for a certain layer $k$ with its lower surface at $x_{3}=z_{k}^{+}(k=1,2, \cdots, N)$, it follows from Eqs. (41) and (43) that the unknown vectors $\mathbf{K}_{1}$ and $\mathbf{K}_{2}$ can be expressed in terms of the generalized displacement and traction vectors as well as the temperature and normal heat flux at its lower surface $x_{3}=z_{k}^{+}$as

$$
\begin{aligned}
& {\left[\begin{array}{c}
\mathbf{K}_{1} \\
\mathbf{K}_{2}
\end{array}\right]=\left\langle e^{\left.-s_{\alpha} z_{k}\right\rangle}\left[\begin{array}{cc}
-\mathbf{B}_{2}^{T} & \mathbf{A}_{2}^{T} \\
\mathbf{B}_{1}^{T} & -\mathbf{A}_{1}^{T}
\end{array}\right]\left[\begin{array}{l}
\mathbf{u} \\
\mathbf{t}
\end{array}\right]_{z_{k}^{+}}-\left\langle e^{-s_{\alpha} z_{k}}\right\rangle\right.} \\
& \times\left[\begin{array}{cc}
-\mathbf{B}_{2}^{T} & \mathbf{A}_{2}^{T} \\
\mathbf{B}_{1}^{T} & -\mathbf{A}_{1}^{T}
\end{array}\right]\left[\begin{array}{cc}
\mathbf{c}_{1} & \mathbf{c}_{2} \\
\mathbf{d}_{1} & \mathbf{d}_{2}
\end{array}\right]\left[\begin{array}{cc}
-f_{1} g_{2} & f_{1} f_{2} \\
f_{2} g_{1} & -f_{1} f_{2}
\end{array}\right]\left[\begin{array}{c}
T \\
q_{3}
\end{array}\right]_{z_{k}^{+}}
\end{aligned}
$$

Then the generalized displacement and traction vectors at any position within this layer are related to the generalized displacement and traction vectors as well as the temperature and normal heat flux at the lower surface $x_{3}=z_{k}^{+}$as follows

$$
\left[\begin{array}{l}
\mathbf{u} \\
\mathbf{t}
\end{array}\right]=\mathbf{E}_{k}\left(x_{3}-z_{k}\right)\left[\begin{array}{l}
\mathbf{u} \\
\mathbf{t}
\end{array}\right]_{z_{k}^{+}}+\mathbf{S}_{k}\left(x_{3}-z_{k}\right)\left[\begin{array}{c}
T \\
q_{3}
\end{array}\right]_{z_{k}^{+}},
$$

where

$$
\begin{aligned}
& \mathbf{E}_{k}\left(x_{3}\right)=\left[\begin{array}{ll}
\mathbf{A}_{1} & \mathbf{A}_{2} \\
\mathbf{B}_{1} & \mathbf{B}_{2}
\end{array}\right]\left\langle e^{s_{\alpha} x_{3}}\right\rangle\left[\begin{array}{cc}
-\mathbf{B}_{2}^{T} & \mathbf{A}_{2}^{T} \\
\mathbf{B}_{1}^{T} & -\mathbf{A}_{1}^{T}
\end{array}\right], \\
& \mathbf{S}_{k}\left(x_{3}\right)=\left[\begin{array}{ll}
\mathbf{c}_{1} & \mathbf{c}_{2} \\
\mathbf{d}_{1} & \mathbf{d}_{2}
\end{array}\right]\left[\begin{array}{cc}
-f_{1} g_{2} e^{\eta_{1} x_{3}} & f_{1} f_{2} e^{\eta_{1} x_{3}} \\
f_{2} g_{1} e^{-\eta_{1} x_{3}} & -f_{1} f_{2} e^{-\eta_{1} x_{3}}
\end{array}\right] \\
& -\mathbf{E}_{k}\left(x_{3}\right)\left[\begin{array}{ll}
\mathbf{c}_{1} & \mathbf{c}_{2} \\
\mathbf{d}_{1} & \mathbf{d}_{2}
\end{array}\right]\left[\begin{array}{cc}
-f_{1} g_{2} & f_{1} f_{2} \\
f_{2} g_{1} & -f_{1} f_{2}
\end{array}\right],
\end{aligned}
$$

Apparently $\mathbf{E}_{k}(0)$ is an $8 \times 8$ identity matrix, and $\mathbf{S}_{k}(0)$ is an $8 \times 2$ zero matrix.

Now that Eqs. (47) and (50) can be concisely written together as follows $\left[\begin{array}{c}\mathbf{u} \\ \mathbf{t} \\ T \\ q_{3}\end{array}\right]=\mathbf{Y}_{k}\left(x_{3}-z_{k}\right)\left[\begin{array}{c}\mathbf{u} \\ \mathbf{t} \\ T \\ q_{3}\end{array}\right]_{z_{k}^{+}}$,

where

$\mathbf{Y}_{k}\left(x_{3}\right)=\left[\begin{array}{cc}\mathbf{E}_{k}\left(x_{3}\right) & \mathbf{S}_{k}\left(x_{3}\right) \\ \mathbf{0}_{2 \times 8} & \mathbf{P}_{k}\left(x_{3}\right)\end{array}\right]$.

is called the layer transfer matrix for the piezothermoelastic problem of layer $k$.

To handle the imperfect interface, we now introduce the interface transfer matrix. Actually by using the boundary conditions Eqs. (10)-(14) on the imperfect interface, the solution at $x_{3}=z_{k}^{+}$can be related to that at $x_{3}=z_{k}^{-}$through the following propagating relation

$\left[\begin{array}{c}\mathbf{u} \\ \mathbf{t} \\ T \\ q_{3}\end{array}\right]_{z_{k}^{+}}=\mathbf{Z}_{k}\left[\begin{array}{c}\mathbf{u} \\ \mathbf{t} \\ T \\ q_{3}\end{array}\right]_{z_{k}^{-}}$,

where the $10 \times 10$ matrix $\mathbf{Z}_{k}$ defined below is the so-called interface transfer matrix for interface $x_{3}=z_{k}$

$\mathbf{Z}_{k}=\left[\begin{array}{ccc}\mathbf{I}_{4 \times 4} & \mathbf{Z}_{12} & \mathbf{0}_{4 \times 2} \\ \mathbf{Z}_{21} & \mathbf{I}_{4 \times 4} & \mathbf{0}_{4 \times 2} \\ \mathbf{0}_{2 \times 4} & \mathbf{0}_{2 \times 4} & \mathbf{Z}_{33}\end{array}\right]$,

with $\mathbf{Z}_{12}$ and $\mathbf{Z}_{21}$ two $4 \times 4$ matrices, and $\mathbf{Z}_{33}$ a $2 \times 2$ matrix.

More specifically if the interface is thermally weakly conducting, then

$\mathbf{Z}_{33}=\left[\begin{array}{cc}1 & -\beta_{T}^{(k)} \\ 0 & 1\end{array}\right]$.

On the other hand if the interface is thermally highly conducting, then

$\mathbf{Z}_{33}=\left[\begin{array}{cc}1 & 0 \\ -\gamma_{T}^{(k)}\left(p^{2}+q^{2}\right) & 1\end{array}\right]$.

If the interface is mechanically compliant and dielectrically weakly conducting, then

$\mathbf{Z}_{12}=\operatorname{diag}\left[\begin{array}{llll}\alpha_{1}^{(k)} & \alpha_{2}^{(k)} & \alpha_{3}^{(k)} & -\beta_{D}^{(k)}\end{array}\right], \mathbf{Z}_{21}=\mathbf{0}_{4 \times 4}$

On the other hand if the interface is mechanically compliant and dielectrically highly conducting, then

$\mathbf{Z}_{12}=\operatorname{diag}\left[\alpha_{1}^{(k)} \quad \alpha_{2}^{(k)} \alpha_{3}^{(k)} \quad 0\right], \mathbf{Z}_{21}=\operatorname{diag}\left[\begin{array}{llll}0 & 0 & 0 & -\gamma_{D}^{(k)}\left(p^{2}+q^{2}\right)\end{array}\right]$.

It is observed that when the interface $x_{3}=z_{k}$ is thermally or dielectrically highly conducting, the interface transfer matrix $\mathbf{Z}_{k}$ is dependent on the values of $p$ and $q$. It can be easily checked that $\mathbf{Z}_{k}$ is an identity matrix when the interface $x_{3}=z_{k}$ is perfect. Consequently, the solution at the top 
surface $x_{3}=H$ of the layered plate can be expressed by that at the bottom surface $x_{3}=0$ of the layered plate as

$\left[\begin{array}{c}\mathbf{u} \\ \mathbf{t} \\ T \\ q_{3}\end{array}\right]_{H}=\boldsymbol{\Omega}\left[\begin{array}{c}\mathbf{u} \\ \mathbf{t} \\ T \\ q_{3}\end{array}\right]_{0}$,

where the $10 \times 10$ matrix $\Omega$ is determined by

$\boldsymbol{\Omega}=\left[\begin{array}{cc}\Xi & \boldsymbol{\Psi} \\ \mathbf{0}_{2 \times 8} & \boldsymbol{\Phi}\end{array}\right]=\mathbf{Y}_{N}\left(h_{N}\right) \times \mathbf{Z}_{N} \times \mathbf{Y}_{N-1}\left(h_{N-1}\right)$

$\times \mathbf{Z}_{N-1} \times \cdots \times \mathbf{Y}_{2}\left(h_{2}\right) \times \mathbf{Z}_{2} \times \mathbf{Y}_{1}\left(h_{1}\right)$,

with $\boldsymbol{\Xi}$ being an $8 \times 8$ matrix, $\boldsymbol{\Psi}$ an $8 \times 2$ matrix, $\boldsymbol{\Phi}$ a $2 \times 2$ matrix.

There are twenty unknowns in Eq. (60). Once the ten thermo-electro-mechanical boundary conditions Eqs. (7) and (9) on the bottom and top surfaces of the layered plate are imposed, all the unknowns in Eq. (60) can be uniquely determined. To demonstrate this more clearly, Eq. (60) can be equivalently rewritten as follows

$\left[\begin{array}{l}\mathbf{u}(H) \\ \mathbf{t}(H)\end{array}\right]=\Xi\left[\begin{array}{l}\mathbf{u}(0) \\ \mathbf{t}(0)\end{array}\right]+\Psi\left[\begin{array}{c}T(0) \\ q_{3}(0)\end{array}\right]$,
$\left[\begin{array}{c}T(H) \\ q_{3}(H)\end{array}\right]=\boldsymbol{\Phi}\left[\begin{array}{c}T(0) \\ q_{3}(0)\end{array}\right]$.

By imposing the thermal boundary conditions Eqs. (7) and $(9)_{5}$, the temperature and normal heat flux at the two surfaces of the layered plate can be uniquely determined as

$$
\left[\begin{array}{c}
T(0) \\
q_{3}(0) \\
T(H) \\
q_{3}(H)
\end{array}\right]=\left[\begin{array}{cccc}
k_{33}^{(1)} h_{a} & 1 & 0 & 0 \\
0 & 0 & k_{33}^{(N)} h_{b} & -1 \\
\Phi_{11} & \Phi_{12} & -1 & 0 \\
\Phi_{21} & \Phi_{22} & 0 & -1
\end{array}\right]^{-1}\left[\begin{array}{c}
k_{33}^{(1)} h_{a} T_{a}^{*} \\
k_{33}^{(N)} h_{b} T_{b}^{*} \\
0 \\
0
\end{array}\right]
$$

where the superscripts (1) and $(N)$ refer to the thermal conductivity coefficients of the bottom and top layers, respectively; $\boldsymbol{\Phi}_{i j}(i, j=1,2)$ are the components of $\boldsymbol{\Phi}$.

Consequently by imposing the electromechanical boundary conditions Eqs. (7) $)_{1-4}$ and (9) $1-4$, the generalized displacement and traction vectors at the two surfaces of the layered plate can be uniquely determined. We present the results below for four different combinations of the electric loads on the two surfaces of the layered plate.

- The normal electric displacement is given on the two surfaces of the layered plate:

$\mathbf{t}(0)=\left[\begin{array}{llll}\sigma_{13}^{0} & \sigma_{23}^{0} & \sigma_{33}^{0} & D_{3}^{0}\end{array}\right]^{T}$,

$\mathbf{t}(H)=\left[\begin{array}{llll}\sigma_{13}^{H} & \sigma_{23}^{H} & \sigma_{33}^{H} & D_{3}^{H}\end{array}\right]^{T}$,

$\mathbf{u}(0)=\Xi_{21}^{-1} \mathbf{t}(H)-\Xi_{21}^{-1} \Xi_{22} \mathbf{t}(0)-\Xi_{21}^{-1} \boldsymbol{\psi}_{21} T(0)-\Xi_{21}^{-1} \boldsymbol{\psi}_{22} q_{3}(0)$,

$\mathbf{u}(H)=\Xi_{11} \Xi_{21}^{-1} \mathbf{t}(H)+\left(\Xi_{12}-\Xi_{11} \Xi_{21}^{-1} \Xi_{22}\right) \mathbf{t}(0)+$

$\left(\boldsymbol{\psi}_{11}-\Xi_{11} \Xi_{21}^{-1} \boldsymbol{\psi}_{21}\right) T(0)+\left(\boldsymbol{\psi}_{12}-\Xi_{11} \Xi_{21}^{-1} \boldsymbol{\Psi}_{22}\right) q_{3}(0)$, where $\boldsymbol{\Xi}_{i j}(i, j=1,2)$ are $4 \times 4$ block matrices of $\boldsymbol{\Xi} ; \boldsymbol{\Psi}_{i j}(i, j=1,2)$ are $4 \times 1$ block matrices of $\Psi$.

- The electric potential is given on the two surfaces of the layered plate:

$\tilde{\mathbf{t}}(0)=\left[\begin{array}{llll}\sigma_{13}^{0} & \sigma_{23}^{0} & \sigma_{33}^{0} & \phi^{0}\end{array}\right]^{T}$,

$\tilde{\mathbf{t}}(H)=\left[\begin{array}{llll}\sigma_{13}^{H} & \sigma_{23}^{H} & \sigma_{33}^{H} & \phi^{H}\end{array}\right]^{T}$,

$\tilde{\mathbf{u}}(0)=\tilde{\boldsymbol{\Xi}}_{21}^{-1} \tilde{\mathbf{t}}(H)-\tilde{\boldsymbol{\Xi}}_{21}^{-1} \tilde{\boldsymbol{\Xi}}_{22} \tilde{\mathbf{t}}(0)-\tilde{\boldsymbol{\Xi}}_{21}^{-1} \tilde{\mathbf{\Psi}}_{21} T(0)-\tilde{\boldsymbol{\Xi}}_{21}^{-1} \tilde{\mathbf{\Psi}}_{22} q_{3}(0)$,

$\tilde{\mathbf{u}}(H)=\tilde{\Xi}_{11} \tilde{\Xi}_{21}^{-1} \tilde{\mathbf{t}}(H)+\left(\tilde{\boldsymbol{\Xi}}_{12}-\tilde{\boldsymbol{\Xi}}_{11} \tilde{\boldsymbol{\Xi}}_{21}^{-1} \tilde{\boldsymbol{\Xi}}_{22}\right) \tilde{\mathbf{t}}(0)+$

$\left(\tilde{\boldsymbol{\Psi}}_{11}-\tilde{\boldsymbol{\Xi}}_{11} \tilde{\boldsymbol{\Xi}}_{21}^{-1} \tilde{\boldsymbol{\Psi}}_{21}\right) T(0)+\left(\tilde{\boldsymbol{\Psi}}_{12}-\tilde{\boldsymbol{\Xi}}_{11} \tilde{\boldsymbol{\Xi}}_{21}^{-1} \tilde{\boldsymbol{\Psi}}_{22}\right) q_{3}(0)$,

where

$\tilde{\mathbf{u}}=\left[\begin{array}{llll}u_{1} & u_{2} & u_{3} & D_{3}\end{array}\right]^{T}$,

and

$$
\begin{aligned}
& {\left[\begin{array}{ll}
\tilde{\boldsymbol{\Xi}}_{11} & \tilde{\boldsymbol{\Xi}}_{12} \\
\tilde{\boldsymbol{\Xi}}_{21} & \tilde{\boldsymbol{\Xi}}_{22}
\end{array}\right]=\left[\begin{array}{ll}
\mathbf{J}_{1} & \mathbf{J}_{2} \\
\mathbf{J}_{2} & \mathbf{J}_{1}
\end{array}\right]\left[\begin{array}{ll}
\boldsymbol{\Xi}_{11} & \boldsymbol{\Xi}_{12} \\
\boldsymbol{\Xi}_{21} & \boldsymbol{\Xi}_{22}
\end{array}\right]\left[\begin{array}{ll}
\mathbf{J}_{1} & \mathbf{J}_{2} \\
\mathbf{J}_{2} & \mathbf{J}_{1}
\end{array}\right],} \\
& {\left[\begin{array}{ll}
\tilde{\mathbf{\Psi}}_{11} & \tilde{\boldsymbol{\Psi}}_{12} \\
\tilde{\boldsymbol{\Psi}}_{21} & \tilde{\boldsymbol{\Psi}}_{22}
\end{array}\right]=\left[\begin{array}{ll}
\mathbf{J}_{1} & \mathbf{J}_{2} \\
\mathbf{J}_{2} & \mathbf{J}_{1}
\end{array}\right]\left[\begin{array}{ll}
\boldsymbol{\Psi}_{11} & \boldsymbol{\Psi}_{12} \\
\boldsymbol{\Psi}_{21} & \boldsymbol{\Psi}_{22}
\end{array}\right],} \\
& \mathbf{J}_{1}=\operatorname{diag}[1,1,1,0], \mathbf{J}_{2}=\operatorname{diag}[0,0,0,1] .
\end{aligned}
$$

- The normal electric displacement is given on the top surface, while the electric potential is prescribed on the bottom surface:

$$
\begin{aligned}
& \tilde{\mathbf{t}}(0)=\left[\begin{array}{llll}
\sigma_{13}^{0} & \sigma_{23}^{0} & \sigma_{33}^{0} & \phi^{0}
\end{array}\right]^{T}, \\
& \mathbf{t}(H)=\left[\begin{array}{llll}
\sigma_{13}^{H} & \sigma_{23}^{H} & \sigma_{33}^{H} & D_{3}^{H}
\end{array}\right]^{T}, \\
& \tilde{\mathbf{u}}(0)=\tilde{\Xi}_{21}^{1} \mathbf{t}(H)-\tilde{\Xi}_{21}^{1} \tilde{\Xi}_{22} \tilde{\mathbf{t}}(0)-\tilde{\Xi}_{21}^{1} \Psi_{21} T(0)-\tilde{\Xi}_{21}^{1} \boldsymbol{\Psi}_{22} q_{3}(0), \\
& \mathbf{u}(H)=\tilde{\Xi}_{11} \tilde{\Xi}_{21}^{-1} \mathbf{t}(H)+\left(\tilde{\Xi}_{12}-\tilde{\Xi}_{11} \tilde{\Xi}_{21}^{-1} \tilde{\Xi}_{22}\right) \tilde{\mathbf{t}}(0)+ \\
& \left(\Psi_{11}-\tilde{\Xi}_{11} \tilde{\Xi}_{21}^{-1} \Psi_{21}\right) T(0)+\left(\boldsymbol{\Psi}_{12}-\tilde{\Xi}_{11} \tilde{\Xi}_{21}^{-1} \Psi_{22}\right) q_{3}(0),
\end{aligned}
$$

where

$$
\left[\begin{array}{ll}
\tilde{\boldsymbol{\Xi}}_{11} & \tilde{\boldsymbol{\Xi}}_{12} \\
\tilde{\boldsymbol{\Xi}}_{21} & \tilde{\boldsymbol{\Xi}}_{22}
\end{array}\right]=\left[\begin{array}{ll}
\boldsymbol{\Xi}_{11} & \boldsymbol{\Xi}_{12} \\
\boldsymbol{\Xi}_{21} & \boldsymbol{\Xi}_{22}
\end{array}\right]\left[\begin{array}{ll}
\mathbf{J}_{1} & \mathbf{J}_{2} \\
\mathbf{J}_{2} & \mathbf{J}_{1}
\end{array}\right],
$$

with $\tilde{\mathbf{u}}, \mathbf{J}_{1}$ and $\mathbf{J}_{2}$ being defined in Eqs. (66) and (67).

- The normal electric displacement is given on the bottom surface, while the electric potential is prescribed on the top surface:

$$
\begin{aligned}
& \mathbf{t}(0)=\left[\begin{array}{llll}
\sigma_{13}^{0} & \sigma_{23}^{0} & \sigma_{33}^{0} & D_{3}^{0}
\end{array}\right]^{T}, \\
& \tilde{\mathbf{t}}(H)=\left[\begin{array}{llll}
\sigma_{13}^{H} & \sigma_{23}^{H} & \sigma_{33}^{H} & \phi^{H}
\end{array}\right]^{l}, \\
& \mathbf{u}(0)=\tilde{\Xi}_{21}^{-1} \tilde{\mathbf{t}}(H)-\tilde{\Xi}_{21}^{-1} \tilde{\Xi}_{22} \mathbf{t}(0)-\tilde{\Xi}_{21}^{-1} \tilde{\mathbf{\Psi}}_{21} T(0)-\tilde{\Xi}_{21}^{-1} \tilde{\mathbf{\Psi}}_{22} q_{3}(0), \\
& \tilde{\mathbf{u}}(H)=\tilde{\Xi}_{11} \tilde{\Xi}_{21}^{-1} \tilde{\mathbf{t}}(H)+\left(\tilde{\Xi}_{12}-\tilde{\Xi}_{11} \tilde{\Xi}_{21}^{-1} \tilde{\Xi}_{22}\right) \mathbf{t}(0)+ \\
& \left(\tilde{\mathbf{\Psi}}_{11}-\tilde{\Xi}_{11} \tilde{\Xi}_{21}^{-1} \tilde{\mathbf{\Psi}}_{21}\right) T(0)+\left(\tilde{\mathbf{\Psi}}_{12}-\tilde{\Xi}_{11} \tilde{\Xi}_{21}^{-1} \tilde{\mathbf{\Psi}}_{22}\right) q_{3}(0),
\end{aligned}
$$

where

$$
\begin{aligned}
& {\left[\begin{array}{ll}
\tilde{\boldsymbol{\Xi}}_{11} & \tilde{\boldsymbol{\Xi}}_{12} \\
\tilde{\boldsymbol{\Xi}}_{21} & \tilde{\boldsymbol{\Xi}}_{22}
\end{array}\right]=\left[\begin{array}{ll}
\mathbf{J}_{1} & \mathbf{J}_{2} \\
\mathbf{J}_{2} & \mathbf{J}_{1}
\end{array}\right]\left[\begin{array}{ll}
\boldsymbol{\Xi}_{11} & \boldsymbol{\Xi}_{12} \\
\boldsymbol{\Xi}_{21} & \boldsymbol{\Xi}_{22}
\end{array}\right],} \\
& {\left[\begin{array}{ll}
\tilde{\boldsymbol{\Psi}}_{11} & \tilde{\boldsymbol{\Psi}}_{12} \\
\tilde{\boldsymbol{\Psi}}_{21} & \tilde{\boldsymbol{\Psi}}_{22}
\end{array}\right]=\left[\begin{array}{ll}
\mathbf{J}_{1} & \mathbf{J}_{2} \\
\mathbf{J}_{2} & \mathbf{J}_{1}
\end{array}\right]\left[\begin{array}{ll}
\boldsymbol{\Psi}_{11} & \boldsymbol{\Psi}_{12} \\
\boldsymbol{\Psi}_{21} & \boldsymbol{\Psi}_{22}
\end{array}\right],}
\end{aligned}
$$


with $\tilde{\mathbf{u}}, \mathbf{J}_{1}$ and $\mathbf{J}_{2}$ being defined in Eqs. (66) and (67).

Consequently the generalized displacement and traction vectors as well as the temperature and normal heat flux at any position within the layered plate can be determined by using Eqs. (52) and (54). In addition the in-plane field components can also be easily determined by using Eq. (44) once the generalized displacement and traction vectors as well as the temperature and normal heat flux are known.

\section{NUMERICAL STUDIES}

Here we consider a nine-layered plate of square shape with $L=L_{x}=L_{y}=1 \mathrm{~m}$ and $H=0.1 \mathrm{~m}$. The bottom eight layers are made of graphite-epoxy composite with the fiber orientation $90^{\circ} / 0^{\circ} / 90^{\circ} / 0^{\circ} / 0^{\circ} / 90^{\circ} / 0^{\circ} / 90^{\circ}$ with respect to the $x_{1}$-axis, and the top layer is made of a PZT-5A piezoelectric material. All the layers have the same thickness. The material properties of the graphite-epoxy [4,6] and PZT-5A [4,6] piezoelectric layers are given in Table 1. As in [6], we treat the graphiteepoxy layer as a piezoelectric material with the piezoelectric moduli set equal to zero. Furthermore we focus on the thermal loads by setting

$T=\sin \left(p x_{1}\right) \sin \left(q x_{2}\right)\left({ }^{\circ} \mathrm{C}\right), x_{3}=0$,

$T=0, x_{3}=H$,

$\sigma_{13}^{0}=\sigma_{23}^{0}=\sigma_{33}^{0}=\phi^{0}=\sigma_{13}^{H}=\sigma_{23}^{H}=\sigma_{33}^{H}=\phi^{H}=0$,

with $n=m=1$.

This boundary value problem was numerically studied by $\mathrm{Xu}$ et al. [4] using the state-space formulation with the interface being assumed to be perfect. When the interface is perfect, our results are in complete agreement with those calculated by Xu [4]. For example we demonstrate in Fig. (1) the through-the-thickness variation of the shear stress $\sigma_{13}$ when the interface is assumed to be perfect. Thus the developed formulas based on the pseudo-Stroh formalism and transfer matrix are validated.

Here we are more interested in the influence of the imperfection of the interfaces on the variations of the field variables. More specifically we consider the following mechanically compliant and thermo-electrically weakly conducting interfaces described by

$\beta_{T}^{(j)}=\delta_{1} \frac{H}{k_{33}^{(1)}}, \alpha_{1}^{(j)}=\alpha_{2}^{(j)}=\alpha_{3}^{(j)}=\delta_{2} \frac{H}{c_{33}^{(1)}}, \beta_{D}^{(j)}=\delta_{3} \frac{H}{\varepsilon_{33}^{(1)}},(j=2-9)$

where $\delta_{1}, \delta_{2}$ and $\delta_{3}$ are three dimensionless nonnegative parameters. Fig. (2) shows the distributions of the normal stress $\sigma_{33}$ along the thickness direction for different values of the dimensionless imperfect interface parameter $\delta=\delta_{1}=\delta_{2}=\delta_{3}$. The horizontal variables are fixed at the center $x_{1}=x_{2}=L / 2$. It is observed from Fig. (2) that the magnitude of normal stress is very small for $\delta=0.35$. On the other hand the magnitude is very high when $\delta=10$. As a result the imperfect interface parameters can be properly designed to reduce the thermal stress level. We also notice that the imperfection of the interface can change the nature of the normal stress from compression to tension.
Table 1. Material Properties of the Graphite-Epoxy and PZT-5A

\begin{tabular}{|c|c|c|}
\hline & $0^{\circ}$ Graphite-Epoxy & PZT-5A \\
\hline$c_{11}(\mathrm{GPa})$ & 183.443 & 99.201 \\
\hline$c_{22}(\mathrm{GPa})$ & 11.662 & 99.201 \\
\hline$c_{33}(\mathrm{GPa})$ & 11.662 & 86.856 \\
\hline$c_{12}(\mathrm{GPa})$ & 4.363 & 54.016 \\
\hline$c_{13}(\mathrm{GPa})$ & 4.363 & 50.778 \\
\hline$c_{23}(\mathrm{GPa})$ & 3.918 & 50.778 \\
\hline$c_{44}(\mathrm{GPa})$ & 2.870 & 21.100 \\
\hline$c_{55}(\mathrm{GPa})$ & 7.170 & 21.100 \\
\hline$c_{66}(\mathrm{GPa})$ & 7.170 & 22.593 \\
\hline$e_{31}\left(\mathrm{Cm}^{-2}\right)$ & 0 & -7.209 \\
\hline$e_{32}\left(\mathrm{Cm}^{-2}\right)$ & 0 & -7.209 \\
\hline$e_{33}\left(\mathrm{Cm}^{-2}\right)$ & 0 & 15.118 \\
\hline$e_{24}\left(\mathrm{Cm}^{-2}\right)$ & 0 & 12.322 \\
\hline$e_{15}\left(\mathrm{Cm}^{-2}\right)$ & 0 & 12.322 \\
\hline$\varepsilon_{11}\left(10^{-10} \mathrm{Fm}^{-1}\right)$ & 153.0 & 153.0 \\
\hline$\varepsilon_{22}\left(10^{-10} \mathrm{Fm}^{-1}\right)$ & 153.0 & 153.0 \\
\hline$\varepsilon_{33}\left(10^{-10} \mathrm{Fm}^{-1}\right)$ & 153.0 & 150.0 \\
\hline$\beta_{11}\left(10^{5} \mathrm{NK}^{-1} \mathrm{~m}^{-1}\right)$ & 2.000 & 3.314 \\
\hline$\beta_{22}\left(10^{5} \mathrm{NK}^{-1} \mathrm{~m}^{-1}\right)$ & 3.506 & 3.314 \\
\hline$\beta_{33}\left(10^{5} \mathrm{NK}^{-1} \mathrm{~m}^{-1}\right)$ & 3.506 & 3.261 \\
\hline$p_{3}\left(10^{-6} \mathrm{CK}^{-1} \mathrm{~m}^{-2}\right)$ & 0 & -8.609 \\
\hline$k_{11}\left(\mathrm{WK}^{-1} \mathrm{~m}^{-1}\right)$ & 1.5 & 1.8 \\
\hline$k_{22}\left(\mathrm{WK}^{-1} \mathrm{~m}^{-1}\right)$ & 0.5 & 1.8 \\
\hline$k_{33}\left(\mathrm{WK}^{-1} \mathrm{~m}^{-1}\right)$ & 0.5 & 1.8 \\
\hline
\end{tabular}

\section{CONCLUSIONS}

We have derived exact solutions for three-dimensional, orthotropic, piezothermoelastic, simply-supported and multilayered rectangular plates with imperfect interfaces under 


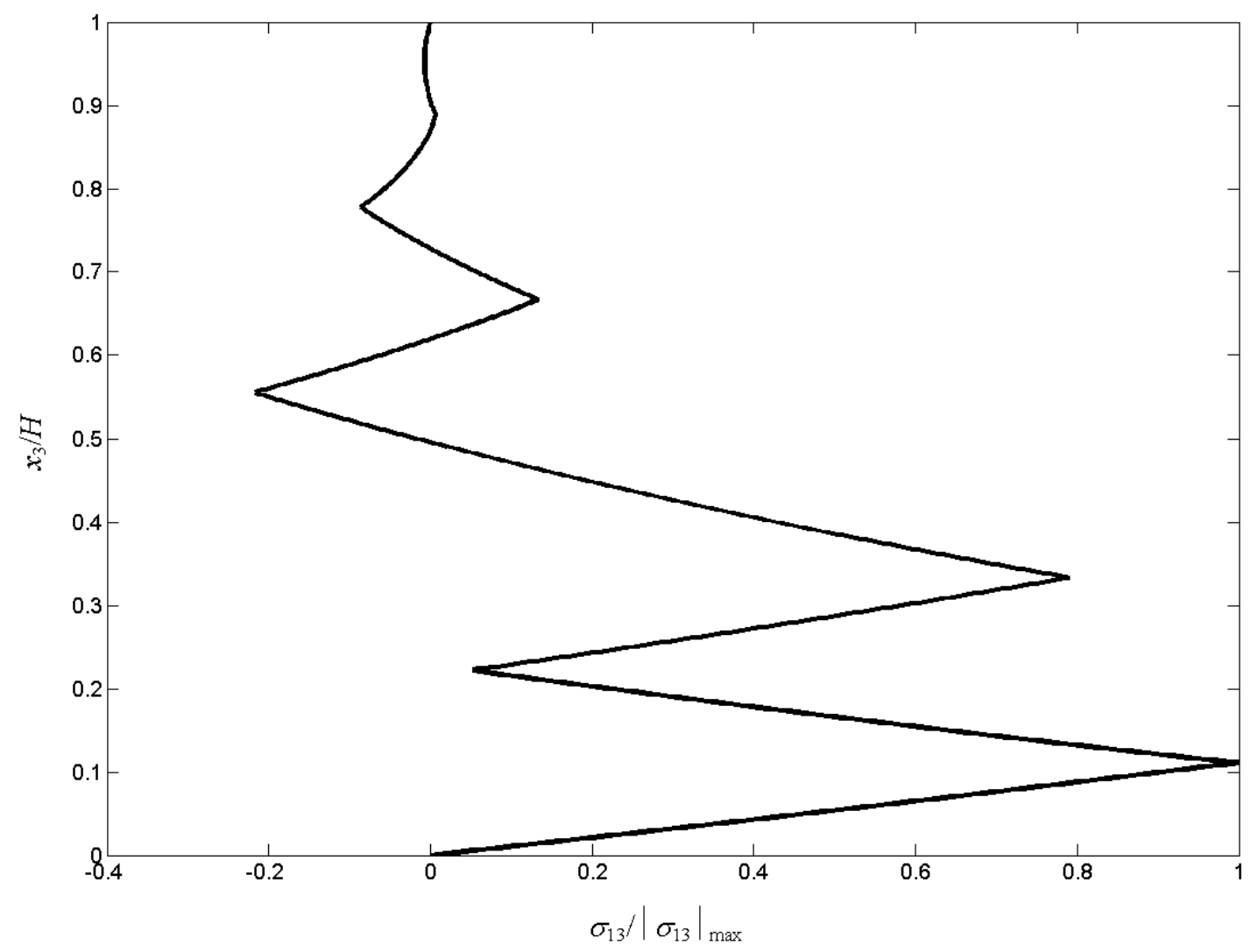

Fig. (1). Through-the-thickness variation of shear stress $\sigma_{13}$ when the interface is assumed to be perfect. Hybrid multilayered plate subjected to temperature change. $L=L_{x}=L_{y}=1 \mathrm{~m}$ and $H=0.1 \mathrm{~m}$.

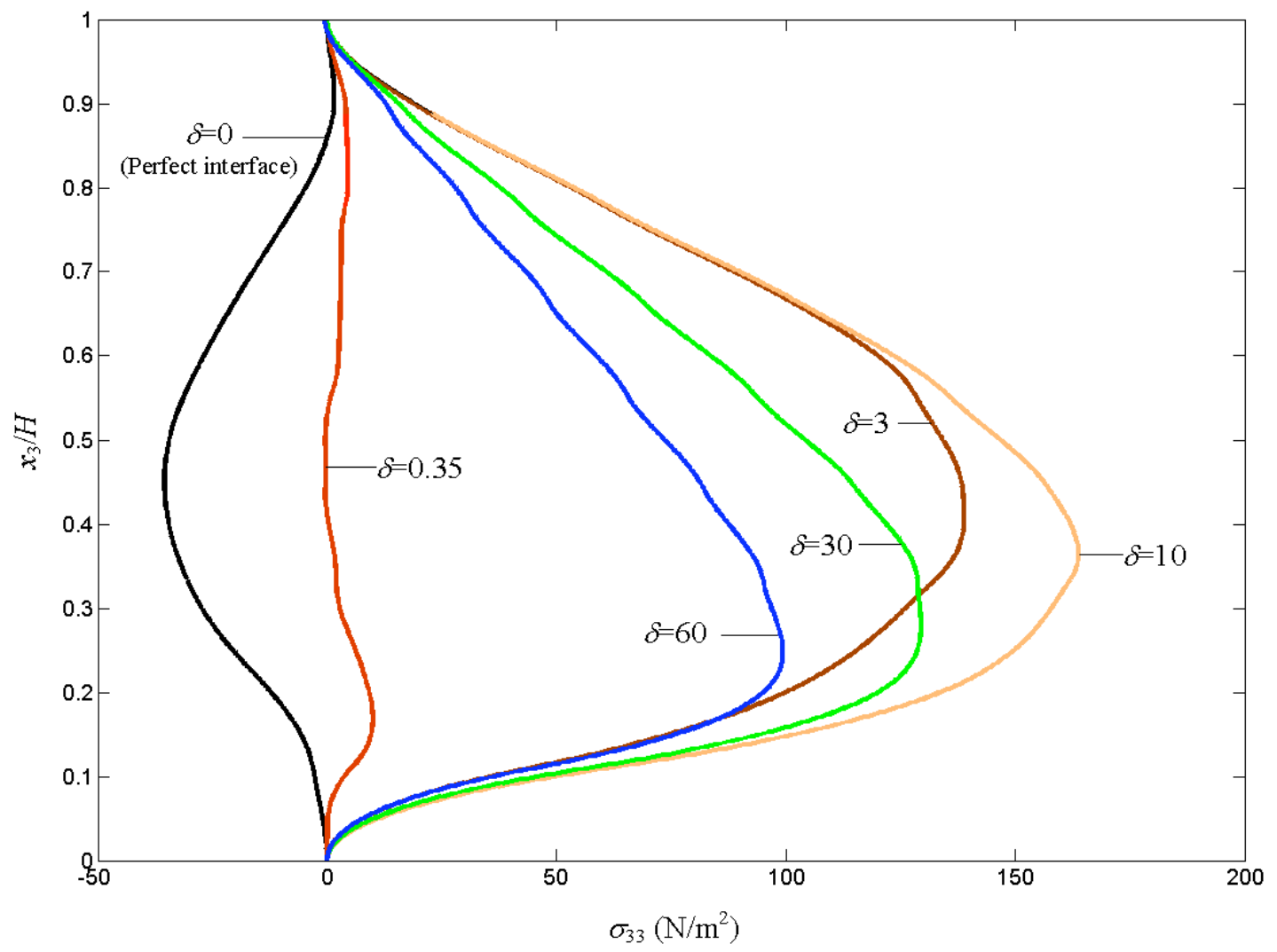

Fig. (2). Distribution of normal stress $\sigma_{33}$ along the thickness direction of the plate for different values of the dimensionless imperfect interface parameter $\delta=\delta_{1}=\delta_{2}=\delta_{3}$. Hybrid multilayered plate subjected to temperature change. $L=L_{x}=L_{y}=1 \mathrm{~m}$ and $H=0.1 \mathrm{~m}$. The horizontal variables are fixed at the center $x_{1}=x_{2}=L / 2$. 
static thermo-electro-mechanical loads. We developed a new and simple formalism that resembles the Stroh formalism so that the solutions in a homogeneous piezothermoelastic layer can be obtained in a concise and elegant form. We also introduced the interface and layer transfer matrices in order to treat the multilayered case with imperfect interface conditions and with the thermal influence. It is found that through introduction of the $10 \times 10$ interface transfer matrix for the general imperfect interface conditions, all imperfect interfaces (e.g., weakly or highly conducting interface) can be discussed within a common framework. Our solutions can also provide benchmarks for various plate theories and numerical methods.

\section{ACKNOWLEDGEMENTS}

This work was partially supported by ARL/ARO \& AFRL/AFOSR. The reviewers' comments on the initial manuscript are highly appreciated.

\section{REFERENCES}

[1] Pagano NJ. Exact solutions for composites in cylindrical bending. J Compos Mater 1969; 3: 398-411.

[2] Pan E. An exact solution for transversely isotropic, simply supported and layered rectangular plates. J Elast 1991; 25: 101-16.

[3] Ray MC, Rao KM, Samanta B. Exact solution for static analysis of intelligent structures under cylindrical bending. Comput Struct 1993; 47: 1031-42.

[4] Xu KM, Noor AK, Tang YY. Three-dimensional solutions for coupled thermoelectroelastic response of multilayered plates. Comput Methods Appl Mech Engrg 1995; 126: 355-71.

[5] Dube GP, Kapuria S, Dumir PC. Exact piezothermoelastic solution of simply-supported orthotropic flat panel in cylindrical bending. Int J Mech Sci 1996; 38: 1161-77.

[6] Vel SS, Batra RC. Three-dimensional analytical solution for hybrid multilayered piezoelectric plates. J Appl Mech 2000; 67: 558-67.
[7] Reddy JN, Cheng ZQ. Three-dimensional solutions of smart functionally graded plates. J Appl Mech 2001; 68: 234-41.

[8] Pan E. Exact solution for simply supported and multilayered magneto-electro-elastic plates. J Appl Mech 2001; 68: 608-18.

[9] Pan E, Han F. Exact solution for functionally graded and layered magneto-electro-elastic plates. Int J Engng Sci 2005; 43: 321-39.

[10] Benveniste Y, Miloh T. The effective conductivity of composite with imperfect contact at constituent interfaces. Int J Engng Sci 1986; 24: 1537-52.

[11] Benveniste Y. On the decay of end effects in conduction phenomena: a sandwich strip with imperfect interfaces of low or high conductivity. J Appl Phys 1999; 86: 1273-79.

[12] Chen T. Thermal conduction of a circular inclusion with variable interface parameter. Int J Solids Struct 2001; 38: 3081-97.

[13] Fan H, Sze KY. A micro-mechanics model for imperfect interface in dielectric materials. Mech Mater 2001; 33: 363-70.

[14] Wang X, Pan E. A moving screw dislocation interacting with an imperfect piezoelectric bimaterial interface. Phys Stat Sol (b) 2007; 224: 1940-56.

[15] Ru CQ, Schiavone P. A circular inclusion with circumferentially inhomogeneous interface in antiplane shear. Proc R Soc Lond A 1997; 453: 2551-72.

[16] Fan H, Wang GF. Screw dislocation interacting with imperfect interface. Mech Mater 2003; 35: 943-53.

[17] Wang X, Zhong Z. Three-dimensional solution of smart laminated anisotropic circular cylindrical shells with imperfect bonding. Int J Solids Struct 2003; 40: 5901-21.

[18] Benveniste Y. A general interface model for a three-dimensional curved thin anisotropic interphase between two anisotropic media. J Mech Phys Solids 2006; 54: 708-34.

[19] Wang X, Pan E, Roy AK. New phenomena concerning a screw dislocation interacting with two imperfect interfaces. J Mech Phys Solids (2007), available from: doi:10.1016/j.jmps.2007.03.017.

[20] Kattis MA, Mavroyannis G. Feeble interfaces in bimaterials. Acta Mech 2006; 185: 11-29.

[21] Chen WQ, Cai JB, Ye GR, Wang YF. Exact three-dimensional solutions of laminated orthotropic piezoelectric rectangular plates featuring interlaminar bonding imperfections modeled by a general spring layer. Int J Solids Struct 2004; 41: 5247-63. 